\title{
Les grandes orientations de l'accord climatique de Paris 2015
}

\author{
Michel Damian ${ }^{1}$, Mehdi Abbas ${ }^{1}$, Pierre Berthaud ${ }^{2}$ \\ 1 Économiste, Université de Grenoble, UMR PACTE, EDDEN, CNRS, 38040 Grenoble, France \\ 2 Économiste, Université de Grenoble, CREG, 38040 Grenoble, France
}

\author{
Mots-clés : \\ CCNUCC; \\ protocole de Kyoto, \\ conférence de Paris \\ 2015 ; contributions \\ nationalement \\ déterminées ; \\ principe de \\ responsabilités \\ communes mais \\ différenciées ; \\ gouvernance
}

\author{
Keywords: \\ United Nations \\ Framework \\ Convention on \\ Climate Change; \\ Paris 2015 Conference; \\ Paris 2015 Agreement; \\ intended nationally \\ determined \\ contributions; \\ common-but- \\ differentiated- \\ responsibilities \\ principle; \\ governance
}

La conférence climatique qui se tiendra à ParisLe Bourget du 30 novembre au 11 décembre 2015 XXI ${ }^{\mathrm{e}}$ Conférence des États signataires, en 1992, de la Convention-cadre des Nations unies sur les changements climatiques (COP 21) - devrait marquer pour des décennies le paysage de la lutte contre le réchauffement et de l'adaptation à celui-ci.
Résumé - L'article est consacré aux grandes orientations, déjà repérables, de l'accord climatique qui sera signé à Paris en décembre 2015, pour devenir effectif à partir de 2020. L'Accord - promu par le G2 États-Unis/Chine - sera fondé sur les seules «politiques nationales »; il tournera le dos à la première politique climatique, celle du protocole de Kyoto, et donc à l'architecture ancienne "par le haut » et à l'ambition d'un accord international contraignant. Des « contributions nationalement déterminées » pour la réduction des émissions de gaz à effet de serre, de nature hétérogène et d'ambitions modestes à moyen terme - et non plus des engagements -, sont attendues de la part de tous les États, y compris ceux inclus par le protocole de Kyoto, comme la Chine, dans la liste des pays en développement. L'accord de Paris devrait constituer un tournant. Il inaugurera une nouvelle gouvernance climatique, dans la continuité de la gouvernance centrée sur les États, mais cette fois à l'échelle globale, c'est-à-dire tenant compte des préférences des 196 parties signataires de la Convention-cadre sur les changements climatiques de 1992 et, en particulier, des plus puissants d'entre eux. On soutient que cet accord, dont il ne faut cependant pas attendre de réduction conséquente des émissions de gaz à effet de serre pour les prochaines décennies, marquera le paysage de la lutte contre le réchauffement et de l'adaptation à celui-ci pour des décennies.

\begin{abstract}
Climate: the key objectives of the Paris 2015 Agreement. The present article focuses on the already discernable key objectives of the climate agreement due to be signed in December 2015 in Paris, to come into force in 2020. The agreement - promoted by the G2 USA-China - will be based exclusively on 'national policies', turning its back on the first climate policy enshrined in the Kyoto Protocol, synonymous with an outdated, top-down architecture and hopes of a binding international agreement. All states, including those, such as China, which the Kyoto Protocol placed in the list of developing countries, are expected to propose 'intended nationally determined contributions' to cutting greenhousegas emissions. These contributions are heterogeneous, with only modest medium-term targets, and not legally binding. The Paris Agreement will represent a turning point, heralding a new climate governance in the continuation of state-centered governance, but henceforth on a global scale. In other words the agreement will take into account the preferences of the 196 parties to the 1992 Framework Convention on Climate Change, in particular those of the most powerful among their number. We maintain that this agreement will change the course of climate change mitigation and adaptation for decades.
\end{abstract}

Auteur correspondant : M. Damian, michel.damian@upmf-grenoble.fr 
milieux économiques, les patrons de quelques-unes des plus grandes firmes multinationales, de très nombreuses ONG, ainsi que des militants de la société civile et des mouvements de justice climatique. La Conférence sera présidée par la France, qui coordonnera les positions de l'Union européenne, avec des enjeux diplomatiques et politiques conséquents.

Le précédent et premier accord climatique à vocation internationale, le protocole de Kyoto, signé en 1997, arrivé à expiration en 2012, prolongé jusqu'à 2020, aura été une impasse : il ne couvre aujourd'hui qu'environ $12 \%$ des émissions mondiales de gaz à effet de serre.

La XXe Conférence climatique (COP 20), tenue à Lima, au Pérou, du $1^{\text {er }}$ au 12 décembre 2014, était censée préparer celle de Paris en 2015. Elle s'est conclue par un court texte de quatre pages de décisions - Lima Call for Climate Action (UNFCCC, 2014a) - au contenu minimaliste pour la plupart des observateurs. À ce texte est joint une annexe de 39 pages, en quelque sorte un mandat pour la négociation de l'accord de Paris. Mais il s'agit seulement d'éléments pour la discussion - Elements for a Draft Negotiating Text (UNFCCC, 2014b) -, rien de plus, sans cap précis, sans choix : une liste de tous les sujets qu'il conviendrait d'aborder, avec chaque fois toutes les options possibles.

L'appel de Lima donne cependant un cadre commun et des lignes directrices pour les négociations de 2015, « un pas en avant» pour l'Union européenne, "une bonne base pour un accord ", énonçait le ministre des Affaires étrangères, Laurent Fabius, au sortir de la Conférence. On soutiendra ici - nos développements ont donc aussi un contenu programmatique - qu'un accord sera signé à Paris. Il s'agira alors, en dehors de la Convention-cadre de 1992, du seul accord climatique international, et certainement du premier accord global signé par tous les États pour les prochaines décennies, $c^{\prime}$ est du moins ce qui est attendu.

La conférence de Paris devrait marquer un triple tournant : 1) un accord sur le climat sera sans doute signé, pour devenir effectif à partir de 2020 ; il s'agira d'un accord-cadre, appelé à durer, avec un examen périodique de ce qui aura été promis et réalisé ; 2) l'accord de Paris sera principalement fondé sur les seules politiques nationales ; il tournera le dos à la première politique climatique, celle du protocole de Kyoto, et donc à l'architecture ancienne " par le haut » et à l'ambition d'un accord international contraignant ; 3) dans le langage climatique, des « contributions nationalement déterminées » (Intended Nationally Determined Contributions, INDCs) sont attendues pour réduire les émissions de gaz à effet de serre ; ces contributions - ce ne seront pas des engagements -, de nature hétérogène et $\mathrm{d}$ 'ambitions modestes à moyen terme, devraient provenir de tous les États, y compris ceux inclus, comme la Chine, par le protocole de Kyoto dans la liste des pays en développement.
Les lignes qui suivent traitent exclusivement de ces grandes orientations. Pour mieux comprendre l'origine, la portée et les enjeux de l'« accord de Paris », on revient - par-delà l'agitation événementielle de la diplomatie climatique - sur le temps long des négociations, depuis les années 1990-1992.

Ces grandes orientations, entérinées par la conférence de Lima, font rupture avec les ambitions antérieures $\mathrm{d}$ 'une politique climatique internationale du type protocole de Kyoto. Mais, durant les douze jours que durera la Conférence, bien d'autres sujets, tous importants, seront à l'agenda. On se limite ici à les mentionner : l'action des villes mondiales pour la réduction des émissions ; les programmes de soutien aux technologies à basse teneur en carbone, aux énergies solaire, éolienne, à la bioénergie, à la capture et au stockage du carbone, voire à l'énergie nucléaire ; la réduction des émissions liées à la déforestation, à la dégradation des forêts ainsi qu'à l'usage des terres; le prix minimal à donner au carbone, pour lequel militent aujourd'hui non seulement les institutions des Nations unies, grandes ONG environnementales et experts, mais également la plupart des grandes firmes, banques et fonds de pension ; enfin, à l'encontre des politiques d'austérité, et pour combattre les crises écologiques et sociales, l'enjeu d'investissements massifs pour la transition énergétique, climatique, et l'accès à l'électricité des personnes qui en sont encore dépourvues, pourrait se glisser dans l'ordre du jour.

Deux questions devraient faire l'objet de débats particulièrement difficiles. $\mathrm{D}^{\prime}$ une part, celle concernant les mesures et politiques des pays en développement, qui pourraient s'arc-bouter sur leur position constante, celle de la responsabilité historique exclusive des pays développés en matière de concentration des émissions dans l'atmosphère et de réchauffement. D'autre part, celle du financement de l'atténuation des émissions, de l'adaptation aux changements climatiques ainsi que des pertes et dommages (c'est-à-dire le coût des impacts inévitables ou irréversibles lorsque l'adaptation atteint ses limites ou n'est plus possible). Le chiffre litigieux est ici celui des " 100 milliards de dollars" par an pour les pays en développement d'ici 2020, mis sur la table, à la dernière minute, par Barack Obama (et Hillary Clinton) lors de la conférence de Copenhague en 2009, pour tenter d'emporter la signature d'un accord de la part des pays émergents. Pour atteindre ce chiffre « rond » à Paris - une condition préalable absolue pour la signature des pays en développement -, il pourrait y avoir un empilement de dons, subventions, prêts et investissements, fournis par des acteurs de nature diverse. Mais il est à peu près sûr que le Sud dans son ensemble considérera que le compte n'y est pas. Il y aura des désillusions. Les récriminations pourraient être âpres. 


\section{Une conférence préemptée par le G2 climatique États-Unis/Chine ?}

Le protocole de Kyoto était fondamentalement d'inspiration américaine, même si les États-Unis s'en sont retirés par la suite: il reprenait les grandes lignes de l'argumentation soumise par le gouvernement des ÉtatsUnis au début de l'année 1997 (USIA, 1997). Les propositions américaines - marché de permis, mécanisme de développement propre, flexibilité - étaient entièrement dominées par les dogmes de l'économie néoclassique, ceux de la théorie des prix et des incitations de marché ${ }^{1}$.

Pour la conférence de Paris, les États-Unis ont été le premier pays à faire connaître, le 12 février 2014, leurs propositions clés : 1) un accord sur des "politiques nationales ", en lieu et place d'engagements internationaux contraignants de type Kyoto et, 2) des «contributions », et non plus des engagements (USA, 2014a). Cette proposition américaine prend ainsi ses distances avec le substrat économiciste qui présidait au design de Kyoto. Elle se place sur un terrain plus politique qui privilégie l'acceptabilité interne de l'accord international.

Ces orientations majeures se sont dessinées au fil des conférences antérieures. En 2002, la conférence de Delhi insistait sur le fait que la politique climatique ne devrait plus être séparée des politiques nationales de développement. Celle de Bali, en 2007, proposait de recentrer la lutte contre les émissions de gaz à effet de serre sur les politiques nationales (NAMAs ou Nationally Appropriate Mitigation Actions dans le langage climatique). La conférence de Copenhague, en 2009, une date charnière, marque, selon la formule d'Olivier Godard, la " grande bifurcation » de la politique climatique (Godard, 2010). La Conférence s'est en effet soldée par l'abandon de la perspective d'un accord international (précisément de la prolongation du protocole de Kyoto au-delà de sa date d'expiration en 2012), au profit d'un basculement sur des politiques nationales minimalistes, les États se limitant à décliner des réductions d'émissions internes. Cela s'est fait sous la conduite des États-Unis et de la Chine, avec le soutien actif des autres membres du groupe BASIC (le Brésil, l'Afrique du Sud et l'Inde), l’Union européenne

\footnotetext{
1 Kyoto a aussi pu être analysé comme le produit d'un compromis entre une position européenne (quotas de réduction) et américaine (des mécanismes flexibles pour réduire l'incertitude sur les coûts et calmer l'industrie) [Hourcade, 2001 ; Aykut, 2014]. Il est possible de considérer que le système de permis négociables était d'inspiration et de construction américaines (Grubb, 2003). La proposition avait été formulée dès juillet 1996, lors de la COP2 tenue à Genève, par le représentant américain Timothy Wirth, copréfacier en 1988 du rapport Harnessing Market Forces to Protect Environment, qui, pour la première fois, recommandait l'établissement d'un système international de marchés de permis pour les gaz à effet de serre.
}

étant tenue à l'écart du deal décisif. L'Europe a cependant obtenu, lors de la conférence de Durban en 2011, 1'acceptation de la prorogation du protocole de Kyoto, et donc la poursuite de son marché de permis carbone, jusqu'en $2020^{2}$.

À Durban a surtout été prise la décision majeure d'élaborer, pour l'horizon 2015, un nouvel accord global, applicable à tous les États signataires de la Convention et devant entrer en vigueur à partir de 2020 - mais avec un échec de l'Union européenne à emporter l'adhésion sur le caractère juridiquement contraignant du futur accord. En novembre 2013, la déclaration finale de la conférence de Varsovie a retenu le terme de « contributions nationalement déterminées » (Intended Nationally Determined Contributions) et non plus celui d'engagements, qui est définitivement rejeté. En décembre 2014, comme nous l'avons déjà indiqué, la conférence de Lima, a réaffirmé le principe de contributions nationales, avec des marges de manœuvre importantes laissées à la discrétion de chaque État.

On notera que cet intense processus diplomatique ne fait pas que déplacer l'objet de la négociation internationale sur le terrain politique. Il marque aussi un recul de la juridicisation, en tout cas du droit international dur, au profit du volontarisme et de la recherche de compromis politiques ${ }^{3}$. Quoi qu'il en soit, les États-Unis ont été (Berthaud et al., 2004), et demeurent, la clé du régime climatique. Les évolutions de forme et de substance de la coopération climatique internationale sont tout sauf contingentes. Elles portent la marque de la préférence des États-Unis. C'est ce qu'ils feront à Paris, avec, cette fois, l'assentiment et certainement le soutien de la Chine, du moins pour les grands axes de la négociation.

L'accord climatique États-Unis/Chine, signé le 12 novembre 2014, préparé depuis plusieurs années par une diplomatie américaine très active, est en quelque sorte une déclaration officielle de leadership, sur les bases voulues par ce G2 - les deux premières puissances économiques, les deux plus gros consommateurs

\footnotetext{
2 L'officialisation de la prolongation de Kyoto est cependant toujours en attente. Le Protocole a été signé par 192 pays (seuls 38 d'entre eux s'étant engagés, en 1997, à réduire leurs émissions) ; pour qu'il soit officiellement prorogé, il faut que les parlements de $75 \%$ au moins des signataires, soit 144 États, aient ratifié cette prorogation. Fin janvier 2015, 23 pays avaient procédé à cette ratification.

3 La forme juridique de l'accord de Paris demeure imprécise : «Un protocole, un instrument légal ou une solution agréée ayant force légale applicable à toutes les parties » (Durban, 2011). On s'acheminerait plutôt vers un accord « contraignant politiquement et moralement, mais pas légalement »(Stern, 2010). La France milite pour l'obtention d'un « accord global contraignant ». Au vu des positions déjà connues des principaux acteurs, l'Union européenne n'est plus en position d'imposer un accord susceptible d'être " contraignant », en tout cas pas « légalement».
} 
d'énergie et les deux premiers émetteurs de gaz à effet de serre au plan mondial ${ }^{4}$.

L'accord contient une formulation au terme de laquelle les deux pays témoignent de leur volonté commune d'aboutir à un "ambitious 2015 agreement », reflétant «the principle of common but differentiated responsibilities and respective capabilities, in light of different national circumstances ». La Chine reconnaît par là qu'elle a aussi des responsabilités, et qu'elle accepte de s'engager sur la base de ses " circonstances nationales ", qu'elle négociera certes avec acharnement. La subtilité langagière contenue dans l'accord États-Unis/Chine - « in light of different national circumstances »-introduit la possibilité d'une différenciation des contributions, et donc aussi la fin de la séparation radicale entre pays développés et pays en développement, pays Annexe 1 et pays non-Annexe $1 \mathrm{du}$ protocole de Kyoto. La formulation américano-chinoise - il convient donc d'en mesurer toute la portée - est reprise en totalité, mot pour mot, dans l'appel de Lima. Celui-ci, tout à fait inscrit dans la nouvelle philosophie ou vision promue par l'accord États-Unis/Chine, ne contient d'ailleurs plus aucune référence à la distinction - omniprésente et décisive lors de toutes les conférences climatiques précédentes - entre pays Annexe 1 et non-Annexe 1.

Les États-Unis et la Chine ont-ils déjà préempté la conférence de Paris 2015 ? Nous en sommes convaincus, du moins pour les grandes lignes de celle-ci. Demeure cependant une inconnue en ce qui concerne la Chine et les grands pays émergents. Depuis 2009, avant chaque conférence climatique, le groupe BASIC fait état de ses analyses, propositions et exigences. Sa position pour celle de Paris ne devrait pas être connue avant l'automne 2015.

\section{Un accord basé sur les « politiques nationales "}

L'action fondée sur les politiques nationales, avec des «contributions nationalement déterminées», marque un retour aux positions originellement défendues par les États-Unis, le Japon, la Russie mais aussi des pays en développement. L'Europe, depuis les premières réunions à la fin de la décennie 1980, puis lors des négociations pour la rédaction de la Convention-cadre sur les changements climatiques en 1991-1992, a toujours eu la

\footnotetext{
4 Le texte de l'accord climatique États-Unis/Chine est consacré pour moitié - ce qui a été peu noté - à des accords de partenariats industriels, technologiques et à des projets de recherches en commun. Le G2 climatique États-Unis/Chine, auquel nous faisons référence, n'est donc pas seulement diplomatique, il a aussi un contenu économique et productif qui n'a pas d'équivalent à l'échelle bilatérale, du moins dans ses ambitions initiales.
}

position la plus volontariste, défendant l'objectif d'une réduction rapide des émissions dans le cadre d'un accord international contraignant.

Les États-Unis, eux, ont toujours marqué leurs réticences à l'encontre d'une approche négligeant les conditions particulières propres à chaque État, tous différents au regard de leur situation économique, démographique, sociale, et du fait de leurs contraintes énergétiques et marges d'action. «Ils critiquaient la proposition de l'Union européenne comme étant une approche "par le haut" (top down) rigide et inéquitable, ne tenant pas compte des différences entre pays en matière de spécificités nationales et de coûts de mise en œuvre. Les États-Unis soutenaient que la Convention devait, à la place, retenir une approche "par le bas" (bottom up), qui encourage une meilleure information, l'adoption de stratégies nationales et de plans d'action nationaux » (Bodansky, 1993, p. 514). Lors des négociations pour la rédaction de la Convention, le Japon avait proposé, en juillet 1991, une approche dite « pledge and review » (promesse et vérification), où chaque pays aurait énoncé ses modalités et ambitions de réductions, celles-ci étant ensuite vérifiées et validées dans le cadre d'une procédure d'évaluation internationale.

À l'époque, l'approche « par le bas » et la soumission japonaise ont été rejetées. Mais ce qui sera retenu à Paris en 2015 est à peu près le décalque de ce concept inaugural de "pledge and review » ou, plus en accord avec les résultats de Lima, "pledge and chat", voire "pledge and see what happens » (Ott et al., 2014, p. 13). Or, au sortir de la signature de la conférence de Rio en 1992, c'est l'architecture par le haut, avec la construction d'un protocole (ce sera celui de Kyoto), qui l'a emporté.

Pourquoi alors un retour aujourd'hui à des politiques nationales? Tout d'abord, ce retour se nourrit de l'échec de Kyoto. Son élargissement à tous les États développés, et à tous les grands émetteurs, s'est révélé impraticable. La plupart des pays ont d'ailleurs commencé à mettre en œuvre des politiques internes, nationales et locales souvent modestes, mais ce sont les intentions et actions déjà entreprises qu'il faut ici retenir - depuis longtemps. Environ $70 \%$ des émissions mondiales de gaz à effet de serre sont aujourd'hui couvertes par des stratégies et législations nationales (Dubash et al., 2013). Aux États-Unis, dès le milieu de la décennie 2000, les États fédérés avaient mis en place des plans de réduction couvrant plus de $50 \%$ du total des émissions de gaz à effet de serre, dix-sept d'entre eux ayant déjà défini leurs propres objectifs de réduction, en général à l'horizon 2020 par rapport à l'année 1990 (Lutsey et Sperling, 2008). Ensuite, l'acceptation de politiques nationales, d'ambitions variables et hétérogènes est manifestement le plus petit dénominateur commun pour parvenir à la signature d'un accord global.

Pour comprendre le retour à des politiques nationales, il y a enfin une dernière raison, plus décisive, celle de l'articulation défectueuse entre l'international et le 
national, manifeste avec l'approche «par le haut » du protocole de Kyoto ${ }^{5}$. L'explication demande de plus longs développements.

Lorsque s'engagent les premières négociations sur le climat, le modèle d'accord que les négociateurs ont en vue est celui de la préservation de la couche d'ozone. Le premier rapport du GIEC (Groupe intergouvernemental d'experts sur l'évolution du climat) sur les réponses à apporter au changement climatique, remis en octobre 1990, a été sur ce plan très prescriptif, le seul format de coopération envisagé est celui de l'accord international : la convention de Vienne et le protocole de Montréal sont cités sept fois dans les six pages qui tracent ce que pourrait être une convention-cadre sur les changements climatiques (IPCC, 1990, pp. 263-268). Mais on se trompe lourdement si l'on croit que le protocole de Montréal a été initialement impulsé par «le haut» pour ensuite s'imposer aux États concernés. C'est l'inverse qui est vrai (Damian, 2012 ; 2014b).

La grande leçon à retenir est que le régime sur les substances qui appauvrissent la couche d'ozone a été préparé et engagé par des actions nationales (interdiction décisive des aérosols aux États-Unis en 1978, suite à des actions des mouvements environnementalistes devant les tribunaux), bien antérieures au protocole de Montréal lui-même. À cela s'ajoute la disponibilité rapide de technologies de substitution, ce qui n'est pas tout à fait le cas en matière de lutte contre les émissions de gaz à effet de serre.

Dans un article de référence sur les régimes environnementaux, John Meyer et ses cosignataires constatent que l'efficacité de ce type de régime est faible et que la question de savoir s'ils résolvent les problèmes environnementaux «n'est pas claire ». La raison se trouve, selon eux, dans « les intérêts figés et limités » des États et aussi de l'ordre mondial (Meyer et al., 1997, pp. 646-647). Pour les États-Unis, Dana Fisher montre de façon tout à fait convaincante que «le niveau national est la base de la construction de la politique climatique » (Fisher, 2004, p. 4 ; également 2006). C'est sur les fondements de politiques nationales que se construisent les accords internationaux d'environnement, du moins pour ceux qui ont des implications économiques sensibles.

\footnotetext{
5 Le cadre «top-down » de Kyoto, sur lequel nous insistons, renvoie cependant à une pratique beaucoup plus complexe des négociations. Les objectifs de réduction des émissions par pays issus de la conférence de Kyoto ont été le résultat de négociations politiques. Même si l'objectif global de $-5,2 \%$ pour les pays Annexe I a fait l'objet d'une communication médiatique importante, l'analyse des négociations montre qu'il a été déterminé dans un deuxième temps seulement, en agrégeant les objectifs nationaux issus des négociations. De ce point de vue, Kyoto témoigne bien aussi d'une articulation entre le national et l'international.
}

Cela ne signifie cependant pas que les accords internationaux et conférences sur le climat soient sans portée : ils ont été et demeurent déterminants pour construire la prise de conscience, impulser politiques et actions à des échelles multiples, évaluer les objectifs et résultats, et maintenir l'agenda à un haut niveau de priorité.

\section{La fin du « mur de Berlin » entre pays développés et en développement}

Le principe 7 de la déclaration de Rio, signée par tous les chefs d'État présents à la Conférence de 1992, a gravé dans le marbre la responsabilité des pays développés : «Étant donné la diversité des rôles joués dans la dégradation de l'environnement mondial, les États ont des responsabilités communes mais différenciées. Les pays développés admettent la responsabilité qui leur incombe dans l'effort international en faveur du développement durable, compte tenu des pressions que leurs sociétés exercent sur l'environnement mondial et des techniques et des ressources financières dont ils disposent. »Ce principe - au cœur du droit international de l'environnement depuis la conférence de Stockholm (1972) et repris dans la Convention sur les changements climatiques de 1992 et le protocole de Kyoto en 1997 - est depuis un quart de siècle la pièce maîtresse à la fois de l'architecture climatique internationale et de la solidarité face aux menaces du réchauffement. La conférence de Paris verra sa rénovation et son actualisation, non pas dans une réécriture, ou une suppression du contenu des articles affirmant ce principe dans les traités internationaux ou dans la Convention, mais dans la pratique, dans le contenu final de l'Accord. Là encore, la marque des États-Unis aura été déterminante.

Les États-Unis sont depuis le début de la négociation de la Convention sur les changements climatiques un « opposant tenace » - «a persistent objector », écrit Daniel Bodansky (1993, pp. 501-502) - au principe de responsabilités communes mais différenciées. À Rio, ils soulignaient ne pas accepter que le principe 7 puisse impliquer de leur part une reconnaissance d'obligations internationales ou « une diminution des responsabilités des pays en développement »(United Nations, 1993,p. 18). Et encore, fin mai 2012, lors d'une réunion préalable à la conférence de Rio+20 sur le développement soutenable, ils marquaient leurs réticences à ce que le document préparatoire se réfère à ce principe (Third World Network, 2012).

Il n'y a donc rien de surprenant à ce qu'ils conditionnent aujourd'hui leur signature d'un futur accord à l'abandon formel de cette approche à deux vitesses. Les propos du négociateur américain, Todd Stern, sont depuis longtemps clairs. Un quart de siècle après les premières négociations, il n'est en effet plus possible, dit-il, de mettre «la Chine et le Tchad » dans le même panier indifférencié des pays en développement (la liste non- 
Annexe 1 du protocole de Kyoto); le «mur de Berlin » entre pays développés et en développement est caduc: la Chine, aujourd'hui premier émetteur mondial de $\mathrm{CO}_{2}$ (mais avec environ $25 \%$ de ses émissions contenus dans les biens qu'elle exporte), a des émissions par tête qui dépassent celles de la France et, d'ici 2020, celles-ci pourraient être de quelque $60 \%$ supérieures à celles des États-Unis (Stern, 2010 ; 2013). Il en va de même pour les économies en développement à forte croissance économique et démographique comme l'Inde, le Brésil, le Mexique, l'Indonésie ou la Thailande.

La soumission américaine de février 2014 pour la conférence de Paris est également sans ambiguïé : «En ce qui concerne les principes de la Convention, qui incluent mais ne se limitent pas aux responsabilités communes mais différenciées et aux capacités respectives, il ne fait aucun doute qu'ils continueront à s'appliquer [...]. Mais nous ne soutiendrons pas une approche à deux vitesses («a bifurcated approach ») pour le nouvel accord, en particulier si elle est fondée sur un regroupement des pays qui avait peut-être un sens en 1992 mais qui n'est clairement ni rationnel ni praticable pour un accord à l'horizon post-2020. »

À Paris, aucun pays ne devrait être exonéré de ses propres responsabilités, c'est du moins ce qui est escompté. Des contributions sont surtout attendues de la part des quinze économies majeures, les plus gros émetteurs des pays développés et grands pays émergents, qui rejettent à eux seuls quelque $75 \%$ des émissions mondiales de gaz à effet de serre (dont environ $55 \%$ pour le seul bloc Chine, États-Unis, Union européenne). Leur volonté et leur action conditionneront l'ambition de l'Accord et les politiques concrètes des années à venir.

Mais, par-delà les économies majeures du Nord et du Sud, ce sont au total 195 États (plus l'UE) qui seront représentés à Paris. L'inclusion du reste des pays en développement, des pays les moins avancés, des petites économies vulnérables et des États insulaires menacés par la hausse du niveau des océans, sera donc déterminante, puisque l'objectif annoncé est de parvenir à un accord universel, avec la signature de tous les États. Paris pourrait alors marquer la réelle globalisation du régime climatique, à condition toutefois que la question du financement et des transferts de technologie trouve un traitement satisfaisant, et que l'Accord répartisse de façon équitable les efforts.

\section{Des contributions de nature variable et hétérogène}

Les propositions de contributions nationalement déterminées des pays sont attendues entre fin mars (pour ceux qui seront « en mesure de le faire », précise l'appel de Lima) et début octobre 2015.
Ces contributions - de nature, d'ampleur et d'échéance variées, et dont le caractère volontaire doit être souligné - ne seront pas nécessairement comparables les unes avec les autres. De rares pays ou zones géographiques annonceront des réductions d'émissions en pourcentage à tel ou tel horizon de temps. Ce sera - $40 \%$ pour l'Union européenne en 2030 par rapport à 1990. Pour les États-Unis, le président Obama a annoncé, lors de l'accord États-Unis/Chine de novembre 2014, une réduction des émissions pour son pays de -26 à $-28 \%$ d'ici 2025 par rapport à l'année de base 2005. La Chine, qui n'avait proposé jusqu'à présent que de réduire son intensité carbone, envisage maintenant, depuis l'accord climatique avec les États-Unis, d'atteindre un pic pour ses émissions de $\mathrm{CO}_{2}$ autour de 2030. Quant à l'Inde, avec 300 millions de personnes sans accès à l'électricité, mais aussi dix des vingt villes les plus polluées de la planète (avec Delhi qui est au moins deux fois plus polluée que Pékin), elle n'entend pas du tout être mise sur le même plan que la Chine et $a$ fortiori les États-Unis (la consommation moyenne d'énergie par habitant en Inde représente tout juste $7 \%$ de celle d'un Américain) ; elle confirmera cependant des plans ambitieux de développement de son parc solaire et devrait seulement s'engager à réduire l'intensité carbone de son PIB (de 20 à $25 \%$ d'ici 2020).

D'autres mesures ou projets d'action pourraient privilégier la réglementation et les standards technologiques, ou encore des politiques portant sur des secteurs industriels particuliers, certainement la voie la plus prometteuse (Barrett, 2010). Des accords sur les HFC (hexofluorocarbones), l'aluminium ou les transports, par exemple, entre les grandes puissances et principaux pays exportateurs, permettraient en effet de gérer de manière coopérative, en tout cas moins conflictuelle, les enjeux omniprésents de compétitivité, avec des réductions négociées et progressives d'émissions, sans recourir à des mesures aux frontières (taxe carbone ou quotas d'importation) impossibles aujourd'hui à mettre en œuvre dans le cadre de la Convention de 1992 (Damian et Abbas, 2007).

Comment mesurer et comparer une telle variété de contributions, pour pouvoir ensuite les additionner dans un accord global de réduction des émissions? Sans clarté, il sera difficile de comprendre les intentions des États, ce qui accroîtra le niveau d'incertitude sur les estimations futures de concentration de gaz à effet de serre dans l'atmosphère. Comment, plus précisément, convertir des contributions de nature différente dans une métrique commune pour pouvoir les comparer les unes aux autres, puis suivre les progrès réalisés ? La conférence de Lima a malheureusement entériné une formulation très floue pour la communication des contributions nationales: aucune obligation précise et commune à toutes les parties; il est seulement mentionné que les 
informations fournies par les États peuvent inclure ( "may include » et non pas «shall », qui a été refusé) des degrés de précision (données quantifiées, années de base, période de mise en œuvre) variés, laissés finalement à l'appréciation de chacun.

Un des enjeux de la conférence de Paris sera donc de dégager un modus operandi pour mesurer et vérifier les émissions. Lors de la conférence climatique de Varsovie, en novembre 2013, les pays présents ont accepté de mesurer les efforts de lutte contre la déforestation tropicale selon des critères à peu près identiques. On pourrait voir là un espoir pour qu'il en aille de même pour la mesure de leurs réductions d'émissions de gaz à effet de serre. Mais les difficultés ne seront pas les mêmes, car la mesure et la vérification vont, cette fois, porter sur des actions variables, sur des gaz à effet de serre qui pourront être différents les uns des autres, sur des secteurs économiques particuliers, sur des politiques et mesures de nature diverse. À Lima, la Chine, tout spécialement, a refusé un mécanisme détaillé et explicite de reporting et vérification des réductions d'émissions, laissant entendre que ce serait un affront à sa souveraineté. Les Nations unies ont finalement été chargées, au terme de Lima, d'évaluer l'effet agrégé de ces contributions pour le $1^{\mathrm{er}}$ novembre 2015, soit un tout petit mois avant le début de la Conférence. Ce n'est donc pas avant les négociations de Paris que les propositions des uns et des autres seront scrutées, évaluées et commentées.

\section{Une nouvelle gouvernance climatique globale}

La nouvelle gouvernance climatique - toujours centrée sur les États, mais cette fois à l'échelle globale, c'està-dire tenant compte des préférences des 195 États signataires de la Convention-cadre, et en particulier des plus puissants d'entre eux - devrait être aux antipodes du régime de Kyoto. Une incursion sur le terrain de la pensée économique est ici nécessaire, pour ne pas occulter les positions des uns et des autres, ni leur part de responsabilité, dans les débats d'idées et plaidoyers pour le climat. On pourra alors mieux comprendre en quoi consistent la gouvernance et la politique climatiques qui sortiront de la conférence de Paris.

L'économie standard de l'environnement et de l'effet de serre - dont l'argumentation a sous-tendu la première politique climatique, l'architecture originelle, la construction du protocole de Kyoto, le recours aux marchés de permis carbone et aux mécanismes de flexibilité - a certainement fait perdre deux décennies à la compréhension de la question climatique ; elle a bloqué, en tout cas peu fait progresser, la réflexion sur les voies concrètes de la décarbonisation (Damian, 2014b). La nouvelle politique climatique témoigne, au regard de la pensée économique, d'un changement de paradigme : elle glisse de la théorie néoclassique des prix et des incitations de marché à l'économie classique de la production, à l'action graduelle des États - politiques publiques de recherche et développement, réglementations, établissement de normes et standards, technologies à basse teneur en carbone -, et donc aussi aux tensions et conflits productifs, sociaux et de redistribution internes qui ne manqueront pas, et au sein desquels la réduction des émissions devra se frayer un chemin.

De ce point de vue, les travaux d'expertise conduits en France semblent bien témoigner de ce glissement d'un cadre analytique à un autre. Le rapport publié en 2003 sous la direction du professeur Roger Guesnerie - Kyoto et l'économie de l'effet de serre -, pour le Conseil d'analyse économique, était sous-tendu par la théorie standard de l'environnement et de l'effet de serre (Guesnerie, 2003). Celui qui s'annonce comme la publication marquante pour la conférence de Paris - le Deep Decarbonization Pathways Project (DDPP), coordonné par l'Institut du développement durable et des relations internationales (IDDRI), dirigé par Laurence Tubiana, et le réseau onusien Sustainable Development Solutions Network, animé par Jeffrey Sachs - est lui, comme la soumission américaine de février 2014, sans substrat explicite de théorie économique (Sachs et Tubiana, 2014). Le DDPP, entrepris à la demande du ministère français des Affaires étrangères, se centre, dans une approche essentiellement technologique, sur les trajectoires nationales, concrètes, de décarbonisation à long terme des quinze plus grands émetteurs mondiaux de gaz à effet de serre ${ }^{6}$.

La caractéristique de l'accord de Paris est qu'il sera là pour durer, certainement un tournant pour tout le

\footnotetext{
$6 \quad$ On ne peut rendre compte ici des débats, mais aussi des partis pris, qui agitent la communauté des économistes et experts sur les actions à conduire. Robert Stavins, un des économistes standards de l'environnement et de l'effet de serre les plus connus, s'est contenté récemment d'une argumentation à grandes enjambées - mais il s'agissait d'un article en direction de la presse populaire - sur l'efficacité exclusive des marchés de permis pour réduire les émissions de dioxyde de carbone aux États-Unis à l'horizon 2050 (Damian, 2014a). Ou encore, dénonçant la «mousse médiatique » de la prochaine conférence de Paris, Christian Gollier, Pierre-André Jouvet, Christian de Perthuis, et le prix Nobel d'économie 2014 Jean Tirole, appelaient à " progresser d'ici à 2020 vers un marché transcontinental du carbone » comme solution déterminante à la lutte contre les changements climatiques (Gollier et al., 2014). On se contentera ici de noter que Thomas Schelling, lui aussi prix Nobel d'économie (2005) invite à penser - et avec lui bien d'autres économistes - "outside the box", à aller au-delà du "carbon pricing », défendant avec force que les grands pays s'engagent sur des politiques de recherche et développement, des actes, des alternatives concrètes.
} 
$\mathrm{XXI} \mathrm{I}^{\mathrm{e}}$ siècle. Dans leur soumission pour la préparation de la Conférence, en février 2014, les États-Unis notaient que l'Accord devait être construit et envisagé pour longtemps: "The agreement per se should be built to last, [...] so that it does not have to be amended every time there are refinements to the details of reporting " (USA, 2014a). Dans leur nouvelle soumission de septembre 2014, ils insistent à trois reprises sur ce point: "The agreement should be built for the long term», «The Paris agreement should [...] be durable in the long term", "The core agreement should be built to last » (USA, 2014b).

Il faudra tout d'abord tenter de maîtriser la croissance des émissions globales sur des décennies - une tâche déjà immense. L'étape suivante - réduire les émissions en valeur absolue, tendre vers le niveau le plus bas possible d'émissions, ne plus augmenter le stock de carbone accumulé dans l'atmosphère - est à l'échelle séculaire.

Progressivement et compte tenu des contraintes sociopolitiques, les contributions des États seront vérifiées, ajustées lors de conférences ultérieures. Elles pourraient progressivement devenir moins modestes, avec des ambitions réévaluées, plus de transparence et de comparabilité. Des formes de coopération entre États pourraient porter, dès la conférence de Paris, sur la réduction des émissions à durée de vie courte (HFC, méthane), mais aussi sur des programmes de coopération technologique. Progressivement pourraient être envisagées aussi des régulations sectorielles (notamment pour le ciment, l'aluminium, les transports aériens).

La nouvelle gouvernance climatique sera réaliste et pragmatique, construite sur les efforts concrets de décarbonisation et sur ce qu'il est raisonnablement possible d'atteindre à l'horizon 2030 ou pour le milieu de ce siècle - une modernisation écologique à petits pas. Les organisateurs et diplomates pourront cependant célébrer le réel succès de la Conférence, puisqu'un accord sera signé, avec un contenu constructif ou du moins acceptable pour les économies majeures. La première politique climatique a longtemps été dominée par un agenda plus «punitif » qu' 'incitatif »: répartir le fardeau d'une réduction contraignante des émissions entre les États ; faire payer, par le biais de permis négociables, les producteurs et consommateurs pour leurs émissions, incités qu'ils seraient - croyait-on - à se tourner rapidement vers des technologies et biens à moindre teneur en carbone (dont la plupart n'étaient d'ailleurs pas immédiatement disponibles). Cela ne s'est pas réalisé au rythme escompté.

La politique climatique qui sortira de la conférence de Paris pourra, elle, être présentée sous un agenda «positif», $l^{\prime}$ « Agenda des solutions ». Les États s'engageront politiquement et moralement à faire, en quelque sorte, leur possible pour aller vers des modes de développement plus soutenables à long terme, mais selon leurs capacités, stade de développement et spécificités propres. Investissements, emploi, qualité de vie, solidarité et coopération sur le long terme pourraient être les maîtres mots d'une communication choisie. En affirmant que la lutte contre le changement climatique et la croissance économique peuvent aller de pair, le récent rapport dirigé par Lord Nicholas Stern et l'ancien président mexicain Felipe Calderon - Better Growth, Better Climate: The New Climate Economy Report - pourrait préfigurer ce type de communication (Stern et Calderon, 2014). Des discours et propositions " positifs » pourraient peut-être aussi contribuer à faire de la politique climatique le catalyseur d'un agenda Nord-Sud renouvelé en matière de développement.

\section{Les chemins infinis de la décarbonisation}

Par-delà la signature d'un accord global, le message final de la $\mathrm{XXI}^{\mathrm{e}}$ Conférence climatique risque cependant d'apparaître mitigé, voire désolant. L'objectif d'une réduction rapide des émissions de gaz à effet de serre au cours des prochaines décennies est inatteignable ${ }^{7}$. Les résultats seront à mille lieues des attentes des pays du Sud, en particulier des plus menacés par le réchauffement, comme des revendications portées par les organisations non gouvernementales et les mouvements de justice climatique. Chaque négociation climatique a sa dramaturgie et ses virulences politiques propres. Celle de Paris n'y échappera pas.

Nous avons soutenu qu'un accord serait conclu à Paris. Mais un accord décevant, non contraignant, ou pas d'accord du tout, serait-ce une catastrophe qui ruinerait tout espoir de «sauver la planète » ? La réponse, selon nous raisonnée au terme de ce texte, est «non». Parce que la politique climatique a déjà tourné, en direction du «bas », en direction de tous les acteurs, et c'est au plus proche des grands émetteurs de gaz à effet de serre et des populations que s'engage, que se joue bien au-delà de ce siècle, ce que l'on pourrait appeler la transition climatique. Jeremy Oppenheim, ancien économiste à la Banque mondiale, consultant du groupe McKinsey et, pour ce qui nous intéresse ici, auteur principal du rapport dirigé par Nicholas Stern, et l'ancien président mexicain Felipe Calderon auquel nous venons de faire référence, indique d'ailleurs, comme avec détachement, qu'un accord à Paris " n'est pas essentiel. Tout ce que nous proposons est conforme aux intérêts économiques

\footnotetext{
$7 \quad$ La question la plus sensible et la plus décisive du maintien d'un stock conséquent de carbone et donc de combustibles fossiles en terre - à hauteur de $80 \%, 50 \%$ et $30 \%$ des réserves respectivement de charbon, gaz et pétrole (McGlade et Ekins, 2015) - demeure non résolue tant elle est conflictuelle (Damian,
} 2013). 
des pays » (cité in Pearce, 2014). Nicholas Stern a luimême énoncé avec force à Lima qu'un traité global juridiquement contraignant n'était "pas nécessaire " à Paris. On est en train de comprendre que les négociations internationales, à elles seules, ne sont pas - et n'ont jamais été - déterminantes et ne peuvent apporter toute la solution à la crise climatique.

Les sociétés, dans leur diversité, sont engagées dans des transitions énergétiques, industrielles et sociales de longue durée. On peut soutenir, comme le consultant de Mckinsey, que cela "est conforme aux intérêts économiques des pays ", mais les tensions et conflits ne manqueront pas. Hors quelque révolution radicale, ou guerre, ou catastrophe de grande ampleur, on ne change pas de mode de développement et de modes de vie - et fondamentalement de régime d'accumulation du capital, parce que c'est bien de cela qu'il s'agit (Abbas, 2010) - en quelques années ou même en quelques décennies. Le réchauffement climatique est essentiellement d'origine anthropique, «plus de la moitié de l'augmentation observée de la température moyenne à la surface du globe entre 1951 et 2010 », écrivent les scientifiques du GIEC dans leur cinquième et dernier rapport publié en 2013 (p. 15). Avec ses impacts différenciés, car il n'y a pas un seul climat global mais une mosaïque de climats, le réchauffement est inéluctable.

L'enjeu est à l'échelle historique, "habiter de façon plus sobre, moins barbare, plus équitable et solidaire la Terre » (Bonneuil, 2014, p. 1-6), à l'atmosphère altérée par les quelque 1500 milliards de tonnes de $\mathrm{CO}_{2}$ que nous y avons déjà déversées. L'histoire dure longtemps, «aussi tragique soit-elle ou reste-t-elle (Badie, 2013, p. 57). Cela vaudra pour les chemins infinis de la décarbonisation.

\section{Références}

Abbas, M., 2010. Économie politique globale des changements climatiques, Grenoble, Presses universitaires de Grenoble.

Aykut, S.C., 2014. Gouverner le climat, construire l'Europe : l'histoire de la création d'un marché du carbone (ETS), Critique Internationale, 62, 39-55.

Badie, B., 2013. Quand l'Histoire commence, Paris, CNRS Éditions.

Barrett, S., 2010. Climate change and international trade: Lessons on their linkage from international environmental agreements. Background paper, $2^{\text {nd }}$ Conference Climate Change, Trade and Competitiveness: Issues for the WTO, 16th, 17th and 18th June, Geneva, The Graduate Institute (online: http://www.wto.org/english/res_e/reser_e/ climate_jun10_e/background_paper6_e.pdf).

Berthaud, P., Cavard, D., Criqui, P., 2004. Le régime international pour le climat: vers la consolidation ou l'effondrement ?, Revue Française d'Économie, 19, 2, 163-185.
Bodansky, D., 1993. The United Nations framework convention on climate change: A commentary, Yale Journal of International Law, 18, 451-558.

Bonneuil, C., 2014. L'Anthropocène et ses lectures politiques, Les Possibles, 3 (online: https://france.attac.org/nospublications/les-possibles/numero-3-printemps-2014/ dossier-l-ecologie-nouvel-enjeu/article/1-anthropoceneet-ses-lectures).

Damian, M., 2014a. Robert Stavins on the carbon-pricing regime, The New York Times, 1 June 2014: dodgy arguments, Economics and Policy of Energy and the Environment, 56, 1, 53-61.

Damian, M., 2014b. La politique climatique change enfin de paradigme, Économie Appliquée, 66, 1, 37-72.

Damian, M., 2013. Mauvaise nouvelle pour le climat et les peuples de l'Amazonie équatorienne : l'abandon du projet Yasuni-ITT de gel du pétrole en terre, Natures Sciences Sociétés, 21, 4, 428-435.

Damian, M., 2012. Repenser l'économie du changement climatique, Économie Appliquée, 65, 2, 9-46.

Damian, M., Abbas, M., 2007. Politique climatique et politique commerciale : le projet français de taxe $\mathrm{CO}_{2}$ aux frontières de l'Europe, Revue de l'Énergie, 58, 578, 221-230.

Dubash, N.K., Hagemann, M., Höhne, N., Upadhyaya, P., 2013. Developments in national climate change mitigation legislation and strategy, Climate Policy, 13, 6, 649-664.

Fisher, D.R., 2004. National Governance and the Global Climate Change Regime, Oxford (UK), Rowman \& Littlefield Publishers.

Fisher, D.R., 2006. Bringing the material back in: Understanding the United States position on climate change, Sociological Forum, 21, 3, 467-494.

GIEC, 2013. Résumé à l'intention des décideurs. Changements climatiques 2013 : les éléments scientifiques. Contribution du Groupe de travail I au Cinquième Rapport d'évaluation du Groupe d'experts intergouvernemental sur l'évolution du climat [sous la direction de Stocker, T.F., Qin, D., Plattner, G.-K., Tignor, M., Allen, S.K., Boschung, J., Nauels, A., Xia, Y., Bex, V., Midgley, P.M.], Cambridge (RU), New York, Cambridge University Press (online: http:// www.developpement-durable.gouv.fr/IMG/pdf/ ONERC_SPM_V3b.pdf).

Godard, O., 2010. La grande bifurcation de la conférence de Copenhague, Les Annales des Mines/Responsabilité $\mathcal{E}$ Environnement, 59, 35-41.

Gollier, C., Jouvet, P.A., De Perthuis, C., Tirole, J., 2014. Conférence sur le climat: à Lima il faut aller plus loin !, Le Monde, 10 décembre.

Grubb, M., 2003.The Economics of the Kyoto Protocol, World Economics, 4, 3, 143-189.

Guesnerie, R. (Ed.) 2003. Kyoto et l'économie de l'effet de serre. Rapport $\mathrm{n}^{\circ} 39$, Conseil d'analyse économique, La Documentation française, Paris.

Hourcade, J.C., 2001. Le climat au risque de la négociation internationale, Le Débat, 113, 136-145.

IPCC, 1990. Climate Change: The IPCC Response Strategies, Working Group III, First Assessment Report (FAR). World Meteorological Organisation/United Nations Environment Program, Intergovernmental Panel on Climate Change. 
Lutsey, N., Sperling, D., 2008. America's bottom-up climate change mitigation policy, Energy Policy, 36, 2, 673-685.

McGlade, C., Ekins, P., 2015. The geographical distribution of fossil fuels unused when limiting global warming to $2{ }^{\circ} \mathrm{C}$, Nature, 517, 7533, 187-190.

Meyer, J.W., Franck, D.J., Hironaka, A., Schofer, E., Tuma, N.B., 1997. The Structuring of a World Environmental Regime, 1870-1990, International Organization, 51, 4, 623-651.

Ott, H., Arens, C., Hermwille, L., Mersmann, F., Obergassel, W., Wang-Helmreich, H., Wehnert, T., 2014. Lima Climate Report - COP20 Moves at Snails' Pace on the Road to Paris. Wuppertal Institute for Climate, Environment and Energy (online: http://wupperinst.org/uploads/tx_wupperinst/ lima-results.pdf).

Pearce, F., 2014. Beyond climate change treaties: "A deal in Paris is not essential", theguardian.com, September 29.

Pialot, D., 2015. Climat : « les scientifiques ne peuvent pas être plus alarmistes », Entretien avec Jean Jouzel, La Tribune, 30 janvier (online: http://www.latribune.fr/entreprisesfinance/industrie/energie-environnement/ 20150130trib7e52b97f5/climat-les-scientifiques-nepeuvent-pas-etre-plus-alarmistes.html).

Sachs, J., Tubiana, L. (Eds.) 2014. Pathways to Deep Decarbonization - 2014 Report. Institute for Sustainable Development and International Relations (IDDRI), Sustainable Development Solutions Network (SDSN), Paris, New-York.

Stern, T.D., 2010. A New Paradigm: Climate Change Negotiations in the Post-Copenhagen Era, U.S. Special Envoy for Climate Change, University of Michigan Law School (online: http:/ /www.state.gov/e/oes/rls/remarks/2010/149429.htm).

Stern, T.D., 2013. The Shape of a New International Climate Agreement, Transcript, U.S. Special Envoy for Climate Change, Chatam House, London (online: http:// www.chathamhouse.org/sites/default/files/public/ Meetings/Meeting\%20Transcripts/221013stern.pdf).
Stern, N., Calderon, F. (Eds.) 2014. Better Growth, Better Climate: The New Climate Economy Report. The Global Commission on the Economy and Climate, New York (online: http:// newclimateeconomy.report/).

Third World Network, 2012. "Common but differentiated responsibilities " under threat, TWN Update on Sustainable Development Conference, Geneva, June 8 (online: http:/ / www.twn.my/title2/sdc2012/sdc2012.120606.htm).

UNFCCC, 2014a. Lima Call for Climate Action, Further advancing the Durban Platform, Draft decision-/CP.XX. United Nations Framework Convention on Climate Change (online: http://unfccc.int/files/meetings/lima_dec_2014/ in-session/application/pdf/cpl14.pdf).

UNFCCC, 2014b. Element for a draft negotiating text, Version 2 of 10 December 2014 at 06:30. United Nations Framework Convention on Climate Change (online: http:/ / unfccc.int/ files/meetings/lima_dec_2014/application/pdf/ auv_cop20_lima_call_for_climate_action.pdf).

United Nations, 1993. Report of the United Nations Conference on Environment and Development, Rio de Janeiro, 2-14 June 1992, A/CONF.151/26/rev.1 (vol.II), Proceedings of the Conference, United Nations, New York.

USA, 2014a. U.S. Submission on Elements of the 2015 Agreement. United Nations Framework Convention on Climate Change (UNFCCC), Bonn (online:

https://unfccc.int/files/documentation/ submissions_from_parties/adp/application/pdf/ u.s._submission_on_elements_of_the_2105_agreement.pdf).

USA, 2014b. U.S. Submission - September 2014. United Nations Framework Convention on Climate Change (UNFCCC), Bonn (online: http://unfccc.int/files/bodies/awg/application/pdf/us_submission_fall_2014_final.pdf).

USIA, 1997. Confronting Climate Change: The Heart of the U.S. Proposal, United States Information Agency, Global Issue, 2, 2, 13-15. 